\title{
Communication among blind, deaf and dumb
}

\section{People}

\author{
B. Buvaneswari ${ }^{1}$, T. Hemalatha ${ }^{2}$, G. Kalaivani ${ }^{3}$, P. Pavithra ${ }^{4}$,
} A. R. Preethisree 5

\author{
${ }^{1}$ Associate Professor, Department of Information Technology, Panimalar Engineering College, Chennai, India \\ 2,3,4,5 Department of Information Technology, Panimalar Engineering College, Chennai, India
}

\begin{abstract}
Now-a-days Science and Technology have made the human world so easy but still some physically and visually challenged people suffer from communication with others. In this project, we are going to propose a new system prototype called communication among Blind, deaf and dumb people.This will helps the disabled people to overcome their difficulties in communicating with some other people with disabilities or normal people. The blind people will communicate through the speakers, the deaf and dumb people will see through it and reply through typing in a terminal.These are all done as an application, so that will be easily understand by the people with disabilities.
\end{abstract}

Keywords- Social interaction, blindness, Granger causality.

\section{INTRODUCTION}

Social cognition is the product of the behavioural and cognitive processes dedicated to build our social world. In humans as well as in other species, many social cues take the form of body language and therefore require vision in order to be understood [1]. For example the imitative behaviour in newborn infants provides an innate foundation for understanding inner states belonging to other persons and for mapping others' actions into actions of their own body [2]. Since vision directly enables the contextual perception of surrounding objects, the absence of vision adversely affects not only language, motor, and cognitive development [3], but also social competence [46] in visually impaired people. For example, since totally blind children lack visual references, they might not be aware of social cues in the form of body gestures while communicating with others. Similarly, low vision children might not correctly express and interpret body language because their imitative behaviours during infancy was prevented due to their degraded vision. As a consequence, visually impaired children often face problems in integrating socially. Preschool-age children with visual disabilities often have difficulty engaging in positive social interactions. Many do not display a full range of play behaviours [4-11], spend more time in solitary play interacting more with adults than with their sighted peers $[6,9,10,1217]$, and are typically unable to demonstrate peer-related social competence [18-20]. This social impairment gives rise to feelings of frustration and fear of interaction, rather than self efficacy and independence, which characterize the social experience of typical children.

\section{RELATED WORK}

\section{Participants}

Six visually impaired ( 3 females, age range: mean age: 42 years old) and eight sighted (4 females, age range: mean age: 40 years old) adults participated in the study. Furthermore, seventeen visually impaired (12 females, mean age: 12 years old) and twelve sighted (4 females, mean age: 13 years old) children aged 6 to 17 participated to the study. Adults and children groups were agedmatched $(t(12)=-0.15 ; \mathrm{P}=0.88 ; \mathrm{t}(27)=0.35 ; \mathrm{P}=0.72)$.

\section{ABBI}

$\mathrm{ABBI}$ is a wearable electronic device positioned on the wrist that produces sounds when moved. It comprises an ABBI bracelet and a mobile phone with an ad-hoc application that allows one to control the sound and the volume. The bracelet and the mobile phone communicate via Bluetooth Low-Energy [23, 28].

\section{Audio Social Interaction Task and Protocol}

The task was performed in a dark room for better tracking movements with the motion captive system. The task required the experimenter to wear $\mathrm{ABBI}$ on the wrist and 
move the arm making three different shapes (straight line, circle and lemniscates symbol) in random order. The participant stood in front of the experimenter and had to follow the experimenter movement that the participant perceived from the moving sound source (i.e. the ABBI bracelet). Each trial lasted 30 seconds, and each shape was performed 5 times in a random order, for a total of 15-20 minutes per participant including breaks. Blind and blindfolded sighted participants performed 15 trials in which the shape produced by the experimenter was known in advance and 6 trials in which it was not

\section{LITERATURE SURVEY}

\section{Multi-modal Interfaces for Interaction- Communication between Hearing and Visually Impaired Individuals: Problems \& Issues}

In this paper the author said one important and challenging problem in human interaction is the communication between blind and deaf individuals. The challenge here involves several cases: (i) first case is a deaf person usually does not speak in order a blind person to hear him/her; (ii) second case is when a blind person speaks a deaf person cannot hear; (iii) third case is when a deaf person makes sign language signs a blind person cannot see them. Thus, this paper presents a study on multi-modal interfaces, issues and problems for establishing communication and interaction between blind and deaf persons. A systemprototype Tyflos- Koufos is proposed in an effort for offering solutions to these challenges.

\section{Design of an Assistive Communication Glove using Combined Sensory Channels}

This paper presents a new design of a wireless sensor glove developed for American Sign Language Finger spelling gesture recognition. Five contact sensors are installed on the glove, in addition to five flex sensors on the fingers and a 3D accelerometer on the back of the hand. Each pair of flex and contact sensors are combined into the same input channel on the BSN node in order to save the number of channels and the installation area. After which, the signal is analyzed and separated back into flex and contact features by software. With electrical contacts and wirings made of conductive fabric and threads, the glove design has become thinner and more flexible. For validation, ASL finger spelling gesture recognition experiments have been performed on signals collected from six speech-impaired subjects and a normal subject. With the new sensor glove design, the experimental results have shown a significant increase in classification accuracy.

\section{Automation of the Arabic Sign Language Recognition}

Interfaces in sign language systems can be categorized as direct-device or vision-based. The direct-device approach uses measurement devices that are in direct contact with the hand such as instrumented gloves, flexion sensors, styli and position-tracking devices. On the other hand, the vision-based approach captures the movement of the singer's hand using a camera that is sometimes aided by making the signer wear a glove that has painted areas indicating the positions of the fingers or knuckles. The main advantage of vision-based systems is that the user isn't encumbered by any complex devices; their main disadvantage, however, is that they require a large amount of computation just to extract the hand position before performing any analysis on the images. In this paper we will discuss only the directed-devise methods.

\section{Design of new digital blind feed forward timing recovery algorithm for linear modulations in digital communication receivers}

Synchronizers are a significant part of digital communication receivers since they are used to estimate the received signal timing. Among these synchronizers, non-data-aided (NDA) or blind synchronizers are even more important due to bandwidth efficiency. In this study, a different approach is investigated to derive new feed forward NDA timing estimators based on Newton algorithm. Two novel estimators with four and two samples per symbol are presented and compared with traditional timing estimators, respectively. One of the main advantages of the second proposed estimator is the ability to control the loop gain automatically. This ability improves the convergence speed and robustness of the design. Simulation results show that the presented estimators outperform the conventional estimators over a noisy channel, especially at small excess bandwidth and low signal to noise ratio.

\section{ACE Assisted Communication for Education: Architecture to support Blind \& Deaf communication}

Deaf and blind students face communication barriers that are constantly present in their daily lives. These barriers arise naturally since the deaf community; the blind community and the rest of the students and teachers use different languages and different channels to communicate. These barriers have a significant impact in the academic, personal and professional development of deaf and blind students. Using automatic tools to assist the fluid communication between people using different languages and different channels of communication might 
significantly promote the social inclusion of disabled students. In this paper we describe the ACE architecture which incorporates Virtual Sign, a translator for sign language and other components to allow for the real time translation between sign and oral languages. This architecture supports any sign language. We expect ACE will provide ways for the fluid communication among deaf people, blind people and those not constrained by these disabilities.

\section{EXISTING SYSTEM}

In existing system Sign Language is the only way of communication for speech impaired people. A the general people unable to understand the sign language, so it becomes very difficult for a speech impaired person to communicate with them. There is no system to communicate speech impaired people.

\section{PROPOSED SYSTEM}

In this project an electronic speaking system and a terminal for typing was developed to ease the communication process of speech impaired people and the speaker is developed for speaking for the visually impaired people .This makes the disabled people to communicate easily without the help of others.

\section{MODULES}

\section{BLIND MODULE}

\section{DEAF MODULE}

\section{DUMB MODULE}

\section{BLIND MODULE}

In older days, they use Braille system to communicate with blind people, to overcome this, we use voice controller system to communicate with the blind people .In this, the blind people can talk through the voice controller to communicate with other people. In another case, we have an app like, the blind people can call or message to the respective person in their contact by drawing their own gestures respective of their contacts.

It is much more useful for the blind people to communicate.

\section{DEAF MODULE}

In this deaf module, we can able to communicate with the deaf people, using the terminal. They can type on whatever they want to say and it will be displayed in the terminal page of other people

\section{DUMB MODULE}

Sign languages are languages that are used by dumb people to express their feeling through manual articulations in combination with non-manual elements. Sign languages are also natural languages in their own grammar and lexicon. ${ }^{[1]}$ Sign languages are not universal as they are not mutually intelligible, ${ }^{[2]}$ although there are also striking similarities among sign languages.

Linguists consider spoken and signed communication to be types of natural language as they both express their own thoughts in their way of communication. Sign language should not be confused with body language, as they are a type of nonverbal communication.

\section{FIGURES AND TABLES}

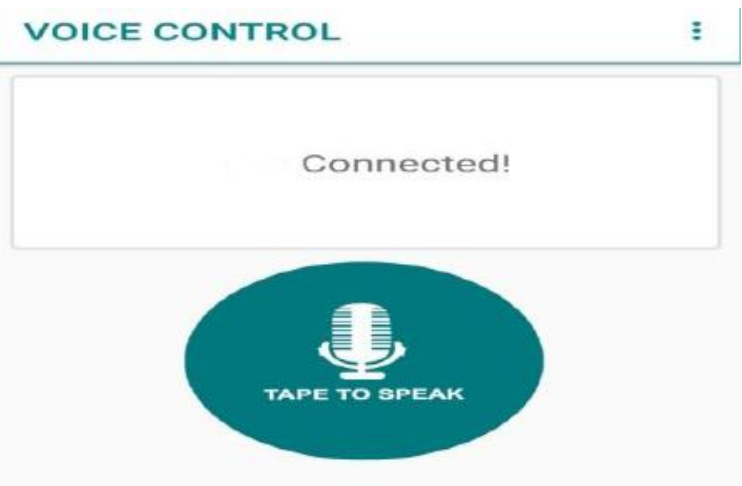

Fig. 1 Blind module

TERMINAL $\vdots$

\section{Enter command here}

Fig. 2 Dumb module

\section{FUTURE ENHANCEMENT}

Future work would utilize a cyber glove and more sophisticated featured extraction algorithm will be utilized. Upon successful implementation of the proposed system, another system would be developed to transfer spoken language into signs using handheld devices like PDA. 


\section{CONCLUSION}

The acquisition of spatial hearing is typically a good indicator of the future ability to independently navigate in the environment and engage in positive social interaction with peers. While for sighted individuals the visual feedback represents the most important incentive for actions and thus for the development of mobility and social skills [32], visually impaired individuals strongly rely on auditory landmarks to encode spatial and social information. Indeed, it has been shown that visually impaired children often have difficulties engaging in positive social interactions, making their assimilation into preschool programs difficult and giving rise to counterproductive feelings of frustration. In this study, we proposed an audio social interaction task in which two agents have to interact thanks to an audio source in order to synchronize their arm movements. As a measure of social competence, the application of the Granger Causality method allows the quantification of sensor motor information flow between partners. This is the first attempt at the evaluation of joint action coordination in visually impaired people. More importantly, we also measured the effects of an extensive but entertaining interventional program with $\mathrm{ABBI}$ on the development of social competence in visually impaired children aged 7-17 years old.

\section{REFERENCES}

[1] M. Groenveld, "Effects of visual disability on behavior and the family," Clinics in developmental medicine, pp. 64-77, 1993.

[2] A. N. Meltzoff and J. Decety, "What imitation tells us about social cognition: a rapprochement between developmental psychology and cognitive neuroscience," Philosophical Transactions of the Royal Society of London B: Biological Sciences, vol. 358, pp. 491500, 2003.

[3] C. Gilbert and H. Awan, "Blindness in children-half of it is avoidable, and suitable cost effective interventions are available," Bmj, vol. 327, pp. 760-761, 2003.

[4] M. J. Guralnick, Assessment of peer relations: Center on Human Development and Disability, University of Washington, 1992.

[5] M. J. Guralnick, R. T. Connor, M. Hammond, J. M. Gottman, and K. Kinnish, "Immediate effects of mainstreamed settings on the social interactions and social integration of preschool children," AJMR-American Journal on Mental Retardation, vol. 100, pp. 359-377, 1996.

[6] M. Rettig, "The play of young children with visual impairments: Characteristics and interventions," Journal of Visual Impairment \& Blindness, 1994.

[7] M. J. Guralnick, R. T. Connor, M. A. Hammond, J. M. Gottman, and K. Kinnish, "The peer relations of preschool children with communication disorders," Child Development, vol. 67, pp. 471489, 1996.

[8] M. J. Guralnick, J. M. Gottman, and M. A. Hammond, "Effects of social setting on the friendship formation of young children differing in developmental status," Journal of Applied Developmental Psychology, vol. 17, pp. 625$651,1996$.

[9] S. Sacks and L. Kekelis, The development of social skills by blind and visually impaired students: Exploratory studies and strategies: Amer Foundation for the Blind, 1992.

[10] S. Sacks, L. Kekelis, and R. Gaylord-Ross, "The Development of Social Skills," New York, NY: American Foundation for the Blind, 1992.

[11] S. R. McConnell and S. L. Odom, "A multi measure performance based assessment of social competence in young children with disabilities," Topics in Early Childhood Special Education, vol. 19, pp. 67-74, 1999.

[12] B. Buvaneswari, Dr. T. Kalpalatha Reddy, 2019, "High Performance Hybrid Cognitive Framework for Bio-Facial Signal Fusion Processing for the Disease Diagnosis", Measurement, ISSN: 0263-2241, Vol. 140, pp.89-99.

[13] B. Buvaneswari, Dr. T. Kalpalatha Reddy, 2019,"ELSA- A Novel Technique to Predict Parkinson's Disease in BioFacial", International Journal of Advanced Trends in Computer Science and Engineering, ISSN 2278-3091, Vol.8, No.1, pp. 12-17.

[14] B. Buvaneswari, Dr. T. Kalpalatha Reddy, 2019, "ACPTAn Intelligent Methodology for Disease Diagnosis", Journal of Advanced Research in Dynamical and Control Systems,

ISSN: 0974-5572, Vol.11, No.4, and pp.2187-2194.

[15] B. Buvaneswari, Dr. T. Kalpalatha Reddy, 2019, "EEG signal classification using soft computing techniques for brain disease diagnosis", Journal of International Pharmaceutical Research, ISSN: 1674-0440, vol.46, No.1, pp.525-528.

[16] B. Buvaneswari, Dr. T. Kalpalatha Reddy, 2017 ," A Review of EEG based Human Facial Expression Recognition Systems in Cognitive Sciences", International Conference on Energy, Communication, Data Analytics and Soft Computing (ICECDS-2017), pp. 462-468 